\title{
Incompressible Limit for the Compressible Flows of Nematic Liquid Crystals in the Whole Space
}

\author{
Young-Sam Kwon \\ Department of Mathematics, Dong-A University, Busan 604-714, Republic of Korea \\ Correspondence should be addressed to Young-Sam Kwon; ykwon@dau.ac.kr
}

Received 30 October 2014; Accepted 24 February 2015

Academic Editor: Mahouton N. Hounkonnou

Copyright (c) 2015 Young-Sam Kwon. This is an open access article distributed under the Creative Commons Attribution License, which permits unrestricted use, distribution, and reproduction in any medium, provided the original work is properly cited.

We consider the compressible flows of liquid crystals arising in a variety of scientific examples. We here study the incompressible limit of weak solutions of the compressible flows of nematic liquid crystals in the whole space $\mathbb{R}^{2}$.

\section{Introduction}

Liquid crystals flows can be found in the natural world and in technological applications with a variety of examples in the area of many proteins, cell membranes and solutions of soap, and various related detergents, as well as the tobacco mosaic virus. We here consider the compressible flows of liquid crystals. Consider the following:

$$
\begin{gathered}
\partial_{t} \varrho+\operatorname{div}_{x}(\varrho \mathbf{u})=0, \\
\partial_{t}(\varrho \mathbf{u})+\operatorname{div}_{x}(\varrho \mathbf{u} \otimes \mathbf{u})+\nabla_{x} \varrho^{\gamma} \\
=\mu \Delta \mathbf{u}-\nu \operatorname{div}_{x}\left(\nabla \mathbf{d} \otimes \nabla \mathbf{d}-\frac{1}{2}|\nabla \mathbf{d}|^{2} \square\right), \\
\partial_{t} \mathbf{d}+\mathbf{u} \cdot \nabla \mathbf{d}=\theta\left(\Delta \mathbf{d}+|\nabla \mathbf{d}|^{2} \mathbf{d}\right),
\end{gathered}
$$

where $\mathbf{u}$ is the vector field, $\varrho$ is the density, $\mathbf{d} \in \mathbb{S}^{1}:=\{\mathbf{d} \in$ $\left.\mathbb{R}^{2}|| \mathbf{d} \mid=1\right\}$ is the macroscopic average of the nematic liquid crystal orientation field, and $\mu, \nu$, and $\theta$ are viscosities. We use the following scalings:

$$
\begin{gathered}
t \approx \frac{t}{\epsilon}, \quad x \approx x, \quad \varrho \approx \varrho_{\epsilon}, \\
\mathbf{u} \approx \epsilon \mathbf{u}_{\epsilon}, \quad \mathbf{d} \approx \mathbf{d}_{\epsilon},
\end{gathered}
$$

and, for the viscosity coefficients,

$$
\mu \approx \epsilon \mu_{\epsilon}, \quad \nu \approx \epsilon^{2} v_{\epsilon}, \quad \theta \approx \epsilon \theta_{\epsilon},
$$

with the convergence of the viscosity coefficients:

$$
\mu_{\epsilon} \longrightarrow \mu>0, \quad v_{\epsilon} \longrightarrow v>0, \quad \theta_{\epsilon} \longrightarrow \theta>0 .
$$

With this scaling, system (1) reads

$$
\begin{gathered}
\partial_{t} \varrho_{\epsilon}+\operatorname{div}_{x}\left(\varrho_{\epsilon} \mathbf{u}_{\epsilon}\right)=0, \\
\partial_{t}\left(\varrho_{\epsilon} \mathbf{u}_{\epsilon}\right)+\operatorname{div}_{x}\left(\varrho_{\epsilon} \mathbf{u}_{\epsilon} \otimes \mathbf{u}_{\epsilon}\right)+\frac{1}{\epsilon^{2}} \nabla_{x} \varrho_{\epsilon}^{\gamma} \\
=\mu_{\epsilon} \Delta \mathbf{u}_{\epsilon}-v_{\epsilon} \operatorname{div}_{x}\left(\nabla \mathbf{d}_{\epsilon} \otimes \nabla \mathbf{d}_{\epsilon}-\frac{1}{2}\left|\nabla \mathbf{d}_{\epsilon}\right|^{2} \square\right), \\
\partial_{t} \mathbf{d}_{\epsilon}+\mathbf{u}_{\epsilon} \cdot \nabla \mathbf{d}_{\epsilon}=\theta_{\epsilon}\left(\Delta \mathbf{d}_{\epsilon}+\left|\nabla \mathbf{d}_{\epsilon}\right|^{2} \mathbf{d}_{\epsilon}\right) .
\end{gathered}
$$

Let us introduce the history of incompressible limit problems. For the case of $\mathbf{d}=0$, incompressible limit problems have been investigated by many authors, starting with the work by Klainerman and Majda [1] for the Euler equations and Lions and Masmoudi [2] for the isentropic Navier-Stokes equations. Similar results in the spirit of the analysis, presented by Lions and Masmoudi [2], are the recent progress by Feireisl and Novotný $[3,4]$ for the full Navier-Stokes Fourier system. For the liquid crystals flows, there is recent progress by Wang and $\mathrm{Yu}[5]$ based on the spectral analysis and Duhamel's principle 
to control difficulties arising in the boundary of bounded domains. In this paper, we will use an abstract result of Kato [6] to show that the energy of acoustic waves decays to zero and this method was used in Feireisl [7] for the full NavierStokes Fourier system in order to derive rigorous limit of low Mach number for compressible flows of nematic liquid crystals in the whole space $\mathbb{R}^{2}$. As an application of this idea, we can also see one work by Kwon and Trivisa [8] for the full magnetohydrodynamic flows.

The outline of this paper is as follows. In Section 1 we present the initial boundary value problem and introduce the notion of weak solutions for the problem. In Section 2 we present the main results of the paper on the low Mach number problem in the whole space. In Section 3 we give the rigorous proof of the low Mach number problem for the flows of nematic liquid crystals in the whole space.

We now introduce the notion of global weak solutions. We first propose the initial data and boundary conditions for the existence of global existence:

$$
\begin{gathered}
\left|\mathbf{d}_{0}\right|=1 \quad \text { a.e. in } \mathbb{R}^{2}, \quad \mathbf{d}_{0}(x)-e_{2} \in W^{1,2}\left(\mathbb{R}^{2}\right), \\
d_{02} \geq \underline{d}_{02},
\end{gathered}
$$

where $e_{2}=(0,1)$ and

$$
(\varrho, \mathbf{u}, \mathbf{d})(t, x) \longrightarrow\left(\varrho_{\infty}, 0, e_{2}\right) \quad \text { as }|x| \longrightarrow \infty .
$$

\subsection{Weak Solutions}

Definition 1. We say that a quantity $\{\varrho, \mathbf{u}, \mathbf{d}\}$ is a weak solution of the compressible flows of liquid crystals (5) supplemented with the initial data $\left\{\varrho_{0}, \mathbf{u}_{0}, \mathbf{d}_{0}\right\}$ provided that the following hold.

(i) The regularity holds:

$$
\begin{gathered}
\varrho \geq 0, \quad \varrho_{\infty} \in C^{0}\left([0, T] ; L_{\text {weak }}^{\gamma}(K)\right) \cap C^{0}\left([0, t] ; L^{p}(K)\right) \\
\cap L_{\text {loc }}^{\gamma+\eta}\left((0, T) \times \mathbb{R}^{2}\right), \\
\mathbf{u} \in L^{2}\left((0, T) ;\left(D^{1,2}\left(\mathbb{R}^{2}\right)\right)^{2}\right) \cap L^{2}\left((0, T) ; L_{\text {loc }}^{2}\left(\mathbb{R}^{2}\right)\right) \\
\varrho \mathbf{u} \in C^{0}\left([0, T] ; L_{\text {weak }}^{2 \gamma /(\gamma+1)}(K)\right) \\
\varrho|\mathbf{u}|^{2} \in L^{\infty}\left((0, T) ; L^{1}\left(\mathbb{R}^{2}\right)\right) \cap L^{2}\left((0, T) ; L_{\text {loc }}^{q}\left(\mathbb{R}^{2}\right)\right) \\
|\mathbf{d}|=1 \quad \text { a.e. in }(0, T) \times \mathbb{R}^{2}, \\
\mathbf{d} \in C^{0}\left([0, T] ; W_{\text {loc }}^{1,2}\left(\mathbb{R}^{2}\right)\right), \\
\partial_{t} \mathbf{d} \in L^{4 / 3}\left(0, T ; L_{\text {loc }}^{2}\left(\mathbb{R}^{2}\right)\right) \\
\nabla \mathbf{d} \in L^{2}\left((0, T) ; W^{1,2}\left(\mathbb{R}^{2}\right)\right) \cap L^{4}\left((0, T) \times \mathbb{R}^{2}\right),
\end{gathered}
$$

where $p \in[1, \gamma), \eta \in(0, \gamma-1), q \geq 1, K \subset \subset \mathbb{R}^{2}$ is an any compact subset of $\mathbb{R}^{2}$, and $\mathbf{u}$ represents a renormalized solution of $(1)$ on $(0, T) \times \mathbb{R}^{2}$; that is, the integral identity

$$
\begin{aligned}
\int_{0}^{T} & \int_{\mathbb{R}^{2}}\left(\varrho B(\varrho) \partial_{t} \varphi+\varrho B(\varrho) \mathbf{u} \cdot \nabla_{x} \varphi-b(\varrho) \operatorname{div}_{x} \mathbf{u} \varphi\right) \mathrm{d} x \mathrm{~d} t \\
\quad & =-\int_{\Omega} \varrho_{0} B\left(\varrho_{0}\right) \varphi(0, \cdot) \mathrm{d} t
\end{aligned}
$$

holds for any test function $\varphi \in \mathscr{D}\left([0, T) \times \mathbb{R}^{2}\right)$ and any $b$ such that

$$
b \in L^{\infty} \cap C[0, \infty), \quad B(\varrho)=B(1)+\int_{1}^{\varrho} \frac{b(z)}{z^{2}} \mathrm{~d} z .
$$

(ii) The balance of momentum holds in distributional sense; namely,

$$
\begin{gathered}
\int_{0}^{T} \int_{\mathbb{R}^{2}}\left(\varrho \mathbf{u} \cdot \partial_{t} \vec{\varphi}+\varrho \mathbf{u} \otimes \mathbf{u}: \nabla_{x} \vec{\varphi}+\frac{1}{\epsilon^{2}} \varrho^{\gamma} \operatorname{div}_{x} \vec{\varphi}\right. \\
\quad+\nu \Pi: \nabla \vec{\varphi}-\mu \nabla \mathbf{u}: \nabla \vec{\varphi}) \mathrm{d} x \mathrm{~d} t \\
=-\int_{\Omega} \varrho_{0} \mathbf{u}_{0} \cdot \vec{\varphi}(0, \cdot) \mathrm{d} x,
\end{gathered}
$$

for any test function $\vec{\varphi} \in \mathscr{D}\left([0, T) ; \mathscr{D}\left(\mathbb{R}^{2} ; \mathbb{R}^{2}\right)\right)$, where $\Pi$ is defined by

$$
\Pi=\nabla \mathbf{d} \otimes \nabla \mathbf{d}-\frac{1}{2}|\nabla \mathbf{d}|^{2} \square .
$$

(iii) The total energy of the system holds as follows:

$E(t)+\int_{0}^{t} \int_{\mathbb{R}^{2}}\left(\mu|\nabla \mathbf{u}|^{2}+\left.\left.\nu \theta|\Delta \mathbf{d}+| \nabla \mathbf{d}\right|^{2} \mathbf{d}\right|^{2}\right) \mathrm{d} x \mathrm{~d} t \leq E(0)$

holds for a.e. $t \in(0, T)$, where

$$
E(t)=\int_{\Omega}\left(\frac{1}{2} \varrho|\mathbf{u}|^{2}+\frac{\varrho^{\gamma}}{\epsilon^{2}(\gamma-1)}+\frac{\nu}{2}|\nabla \mathbf{d}|^{2}\right) \mathrm{d} x,
$$

and we assume $E(0)$.

(iv) The equation of direction field verifies

$$
\begin{aligned}
& \int_{0}^{T} \int_{\mathbb{R}^{2}} \mathbf{d} \cdot \partial_{t} \vec{\varphi} \mathrm{d} x \mathrm{~d} t \\
& \quad+\int_{0}^{T} \int_{\mathbb{R}^{2}}(\mathbf{u} \cdot \mathbf{d}) \operatorname{div}_{x} \vec{\varphi}+(\nabla \mathbf{u} \cdot \mathbf{d}) \cdot \vec{\varphi} \\
& \quad+\theta\left(\Delta \mathbf{d}+|\nabla \mathbf{d}|^{2} \mathbf{d}\right) \cdot \vec{\varphi} \mathrm{d} x \mathrm{~d} t \\
& =-\int_{\mathbb{R}^{2}} \mathbf{d}_{0} \cdot \vec{\varphi}(0, x) \mathrm{d} x
\end{aligned}
$$

for all $\vec{\varphi} \in\left[\mathscr{D}\left([0, T) \times \mathbb{R}^{2}\right)\right]^{2}$. 
Remark 2. There are global weak solutions for compressible flows of nematic liquid crystals in two dimensions and the result is provided in Jiang et al. [9].

We now introduce the weak solutions of the target system.

Definition 3. A couple $\{\mathbf{U}, \mathbf{d}\}$ is said to be a weak solution of the target system of the compressible flows of liquid crystals supplemented with the initial conditions

$$
\mathbf{U}(0, \cdot)=\mathbf{U}_{0}, \quad \mathbf{d}(0, \cdot)=\mathbf{d}_{0},
$$

if the following conditions hold:

(i) $\mathbf{U} \in L^{\infty}\left(0, T ; L_{\text {loc }}^{2}\left(\mathbb{R}^{2} ; \mathbb{R}^{2}\right)\right) \cap L^{2}\left(0, T ; W_{0}^{1,2}\left(\mathbb{R}^{2} ; \mathbb{R}^{2}\right)\right)$,

$$
\operatorname{div}_{x} \mathbf{U}=0 \text { a.e. on }(0, T) \times \mathbb{R}^{2},
$$

and the integral identity

$$
\begin{aligned}
& \int_{0}^{T} \int_{\mathbb{R}^{2}}\left(\mathbf{U} \cdot \partial_{t} \varphi+(\mathbf{U} \otimes \mathbf{U}): \nabla_{x} \varphi-\mu \nabla_{x} \mathbf{U}: \nabla_{x} \varphi\right) \mathrm{d} x \mathrm{~d} t \\
& \quad=-v \int_{0}^{T} \int_{\mathbb{R}^{2}}(\nabla \mathbf{d} \otimes \nabla \mathbf{d}): \nabla \varphi \mathrm{d} x \mathrm{~d} t-\int_{\mathbb{R}^{2}} \mathbf{U} \cdot \varphi(0, \cdot) \mathrm{d} x
\end{aligned}
$$

holds for any test function

$$
\varphi \in \mathscr{D}\left((0, T) \times \mathbb{R}^{2} ; \mathbb{R}^{2}\right), \quad \operatorname{div}_{x} \varphi=0 \text { in } \mathbb{R}^{2} .
$$

(ii) $|\mathbf{d}|=1, \mathbf{d} \in L^{2}\left(0, T ; W^{2,2}\left(\mathbb{R}^{2} ; \mathbb{R}^{2}\right)\right) \cap L^{4}\left((0, T) \times \mathbb{R}^{2}\right)$, and the integral identity

$$
\begin{aligned}
& \int_{0}^{T} \int_{\mathbb{R}^{2}} \mathbf{d} \cdot \partial_{t} \vec{\varphi} \mathrm{d} x \mathrm{~d} t \\
& +\int_{0}^{T} \int_{\mathbb{R}^{2}}\left((\mathbf{U} \cdot \mathbf{d}) \operatorname{div}_{x} \vec{\varphi}+(\nabla \mathbf{U} \cdot \mathbf{d}) \cdot \vec{\varphi}\right. \\
& \left.\quad+\theta\left(\Delta \mathbf{d}+|\nabla \mathbf{d}|^{2} \mathbf{d}\right) \cdot \vec{\varphi}\right) \mathrm{d} x \mathrm{~d} t \\
& =-\int_{\mathbb{R}^{2}} \mathbf{d}_{0} \cdot \vec{\varphi}(0, x) \mathrm{d} x,
\end{aligned}
$$

for all $\vec{\varphi} \in\left[\mathscr{D}\left([0, T) \times \mathbb{R}^{2}\right)\right]^{2}$.

\section{Main Results}

In this section we mention the main result.

Theorem 4. Let $\left\{\varrho_{\epsilon}, \mathbf{u}_{\epsilon}, \mathbf{d}_{\epsilon}\right\}$ be a family of weak solutions to the compressible flow of liquid crystals system in the sense of Definition 1 with $\gamma>1$. Assume the initial condition as follows:

$$
\begin{gathered}
\mathbf{u}_{0, \epsilon} \rightarrow \mathbf{u}_{0} \quad \text { in } L^{2}\left(\mathbb{R}^{2}\right), \quad \varrho_{0, \epsilon} \rightarrow \bar{\varrho} \quad \text { in } L^{2}\left(\mathbb{R}^{2}\right), \\
\mathbf{d}_{0, \epsilon} \rightarrow \mathbf{d}_{0} \quad \text { in } L^{2}\left(\mathbb{R}^{2}\right),
\end{gathered}
$$

$$
\begin{array}{r}
\int_{\Omega}\left(\frac{1}{2} \varrho_{0, \epsilon}\left|\mathbf{u}_{0, \epsilon}\right|^{2}+\frac{1}{2} \nu_{\epsilon}\left|\nabla \mathbf{d}_{0, \epsilon}\right|^{2}\right) d x+\frac{1}{\epsilon^{2}(\gamma-1)} \\
\cdot \int_{\Omega}\left(\varrho_{0, \epsilon}\right)^{\gamma}-\bar{\varrho}^{\gamma}-\gamma \bar{\varrho}^{\gamma-1}\left(\varrho_{0, \epsilon}-\bar{\varrho}\right) d x \leq C .
\end{array}
$$

Then, up to subsequence,

$$
\begin{aligned}
& \varrho_{\epsilon} \longrightarrow \bar{\varrho} \text { a.e.in }(0, T) \times \mathbb{R}^{2}, \\
& \mathbf{u}_{\epsilon} \longrightarrow \mathbf{U} \text { a.e. in }(0, T) \times \mathbb{R}^{2}, \\
& \mathbf{d}_{\epsilon} \longrightarrow \mathbf{d} \text { a.e. in }(0, T) \times \mathbb{R}^{2},
\end{aligned}
$$

where $\{\mathbf{U}, \mathbf{d}\}$ is a weak solution of the incompressible flows of liquid crystals in the sense of Definition 3 with the initial data

$$
\mathbf{U}(0)=\mathbf{P}\left[\mathbf{U}_{0}\right], \quad \mathbf{d}(0, \cdot)=\mathbf{d}_{0},
$$

where Helmholtz's projection $\mathbf{P}=\mathbf{I}-\mathbf{Q}, \mathbf{Q}=\nabla \Delta^{-1} \operatorname{div}$.

\section{Proof of Theorem 4}

In this section we are going to give a rigorous proof of Theorem 4 . To begin with we derive some uniform estimates from the energy inequality.

3.1. Uniform Bounds. In this section we are going to derive some estimates on the sequence $\left\{\varrho_{\epsilon}, \mathbf{u}_{\epsilon}, \mathbf{d}_{\epsilon}\right\}_{\epsilon>0}$. Notice that the function

$$
\varrho \longmapsto \varrho^{\gamma}-\bar{\varrho}^{\gamma}-\gamma \bar{\varrho}^{\gamma-1}(\varrho-\bar{\varrho})
$$

is strictly convex in $[0, \infty)$ and it has global minimum at $\bar{\varrho}$. We now rewrite the energy equality into

$$
\begin{aligned}
\int_{\mathbb{R}^{2}}( & \frac{1}{2} \varrho_{\epsilon}\left|\mathbf{u}_{\epsilon}\right|^{2}+\frac{1}{\epsilon^{2}(\gamma-1)}\left(\varrho_{\epsilon}^{\gamma}-\bar{\varrho}^{\gamma}-\gamma \bar{\varrho}^{\gamma-1}\left(\varrho_{\epsilon}-\bar{\varrho}\right)\right) \\
& \left.+\frac{\nu_{\epsilon}}{2}\left|\nabla \mathbf{d}_{\epsilon}\right|^{2}\right) \mathrm{d} x \\
& +\int_{0}^{t} \int_{\mathbb{R}^{2}}\left(\mu_{\epsilon}\left|\nabla \mathbf{u}_{\epsilon}\right|^{2}+\left.\left.\nu_{\epsilon} \theta_{\epsilon}\left|\Delta \mathbf{d}_{\epsilon}+\right| \nabla \mathbf{d}_{\epsilon}\right|^{2} \mathbf{d}_{\epsilon}\right|^{2}\right) \mathrm{d} x \mathrm{~d} t \\
\leq & E_{0, \epsilon},
\end{aligned}
$$

where

$$
\begin{aligned}
E_{0, \epsilon}=\int_{\mathbb{R}^{2}}( & \frac{1}{2} \varrho_{0, \epsilon}\left|\mathbf{u}_{0, \epsilon}\right|^{2}+\frac{1}{\epsilon^{2}(\gamma-1)} \\
& \cdot\left(\varrho_{0, \epsilon}^{\gamma}-\bar{\varrho}^{\gamma}-\gamma \bar{\varrho}^{\gamma-1}\left(\varrho_{0, \epsilon}-\bar{\varrho}\right)\right) \\
& \left.+\frac{v_{\epsilon}}{2}\left|\nabla \mathbf{d}_{0, \epsilon}\right|^{2}\right) \mathrm{d} x .
\end{aligned}
$$


In accordance with assumptions 4 , we get $E_{0, \epsilon}$ is uniformly bounded for $\epsilon \rightarrow 0$. From the energy inequality (25), we obtain

$$
\begin{gathered}
\text { ess } \sup _{t \in(0, T)}\left\|\sqrt{\varrho_{\epsilon}} \mathbf{u}_{\epsilon}(t)\right\|_{L^{2}\left(\mathbb{R}^{2} ; \mathbb{R}^{2}\right)} \leq C, \\
\text { ess } \sup _{t \in(0, T)}\left\|\nabla \mathbf{d}_{\epsilon}(t)\right\|_{L^{2}\left(\mathbb{R}^{2} ; \mathbb{R}^{2}\right)} \leq C, \\
\text { ess } \sup _{t \in(0, T)}\left\|\left(\Delta \mathbf{d}_{\epsilon}+\left|\nabla \mathbf{d}_{\epsilon}\right|^{2} \mathbf{d}_{\epsilon}\right)(t)\right\|_{L^{2}\left(\mathbb{R}^{2} ; \mathbb{R}^{2}\right)} \leq C, \\
\left\|\nabla_{x} \mathbf{u}_{\epsilon}\right\|_{L^{2}\left((0, T) \times \mathbb{R}^{2}\right)} \leq C, \\
\text { ess } \sup _{t \in(0, T)}\left\|\varrho_{\epsilon}^{\gamma}-\bar{\varrho}^{\gamma}-\gamma \bar{\varrho}^{\gamma-1}\left(\varrho_{\epsilon}-\bar{\varrho}\right)\right\|_{L^{1}\left(\mathbb{R}^{2}\right)} \leq \epsilon^{2} C .
\end{gathered}
$$

For the convenient presentation, we now introduce the set of the essential and residual values:

$$
g=[g]_{\mathrm{ess}}+[g]_{\mathrm{res}},
$$

where $[g]_{\text {ess }}=\chi\left(\varrho_{\epsilon}\right) g,[g]_{\text {res }}=\left(1-\chi\left(\varrho_{\epsilon}\right)\right) g$, and $\chi$ is defined as follows:

$$
\chi(r)= \begin{cases}1, & \forall r \in\left[\frac{\bar{\varrho}}{2}, 2 \bar{\varrho}\right] \\ 0, & \text { otherwise. }\end{cases}
$$

Following the estimate of the fifth line in (27) and the convexity property of function in (24), we get

$$
\begin{aligned}
& \text { ess } \sup _{t \in(0, T)}\left\|\left[\frac{\varrho_{\epsilon}-\bar{\varrho}}{\epsilon}\right]_{\mathrm{ess}}\right\|_{L^{2}\left(\mathbb{R}^{2}\right)} \leq C, \\
& \text { ess } \sup _{t \in(0, T)}\left\|\left[\frac{\varrho_{\epsilon}-\bar{\varrho}}{\epsilon}\right]_{\mathrm{res}}\right\|_{L^{\gamma}\left(\mathbb{R}^{2}\right)} \leq C .
\end{aligned}
$$

We now derive the estimate of the velocity. Notice that

$$
\begin{aligned}
\operatorname{ess} \sup _{t \in(0, T)}\left\|\left[\mathbf{u}_{\epsilon}\right]_{\mathrm{ess}}\right\|_{L^{2}(K)} & \leq C \sup _{t>0}\left(\int_{K} \varrho_{\epsilon}\left|\mathbf{u}_{\epsilon}\right|^{2} \mathrm{~d} x\right)^{1 / 2} \leq C, \\
\left\|\left[\mathbf{u}_{\epsilon}\right]_{\mathrm{res}}\right\|_{L^{2}(K)}^{2} & \leq C \int_{K}\left|\varrho_{\epsilon}-\bar{\varrho}\right|\left|\mathbf{u}_{\epsilon}\right|^{2} \mathrm{~d} x \\
& \leq \epsilon C\left\|\mathbf{u}_{\epsilon}\right\|_{L^{2 p /(p-1)}(K)}^{2} \\
& \leq \epsilon C\left\|\nabla \mathbf{u}_{\epsilon}\right\|_{L^{2}(K)}^{2}
\end{aligned}
$$

with $p=\min \{2, \gamma\}$ and for any $K \subset \subset \mathbb{R}^{2}$ where we have used the Sobolev embedding inequality. Thus we get

$$
\left\|\mathbf{u}_{\epsilon}\right\|_{L^{2}((0, T) \times K)} \leq C,
$$

and the estimates in (32) and (27) imply

$$
\left\|\mathbf{u}_{\epsilon}\right\|_{L^{2}\left(0, T ; H^{1}\left(K ; \mathbb{R}^{2}\right)\right)} \leq C,
$$

for any $K \subset \subset \mathbb{R}^{2}$.

3.2. Convergence of Continuity Equation. For the proof of this section, we will use the uniform estimate (33) to deduce

$$
\mathbf{u}_{\epsilon} \longrightarrow \mathbf{U} \quad \text { weakly in } L^{2}\left(0, T ; W^{1,2}\left(K ; \mathbb{R}^{2}\right)\right),
$$

up to a subsequence of $\{\epsilon>0\}$ and for any $K \subset \subset \mathbb{R}^{2}$. In accordance with (30), we obtain

$$
\varrho_{\epsilon} \longrightarrow \bar{\varrho} \quad \text { in } L^{\infty}\left(0, T ; L^{\gamma}\left(\mathbb{R}^{2}\right)\right)
$$

and so we can take the limit of $\epsilon$ in the continuity equation (9) to get

$$
\int_{0}^{T} \int_{\mathbb{R}^{2}} \mathbf{U} \cdot \nabla_{x} \varphi \mathrm{d} x \mathrm{~d} t=0
$$

for all $\varphi \in C_{c}^{\infty}\left((0, T) \times \mathbb{R}^{2}\right)$.

3.3. Convergence of Moment and Direction Field Equation. This section is devoted to proving the convergence of moment and direction field equation which is based on some uniform estimates of the directional vector fields $\left\{\mathbf{d}_{\epsilon}\right\}_{\epsilon>0}$ in the result of global weak solutions in Jiang et al. [9]. We first use the estimates of (32) and (35) in the previous section to obtain that

$$
\varrho_{\epsilon} \mathbf{u}_{\epsilon} \longrightarrow \bar{\varrho} \mathbf{U}
$$

$$
\text { weakly- }(*) \text { in } L^{\infty}\left(0, T ; L^{2 \gamma / 1+\gamma}\left(K ; \mathbb{R}^{2}\right)\right)
$$

for any $K \subset \subset \mathbb{R}^{2}$. Hence, it follows that

$$
\int_{0}^{T} \int_{\mathbb{R}^{2}} \varrho_{\epsilon} \mathbf{u}_{\epsilon} \otimes \mathbf{u}_{\epsilon}: \nabla_{x} \vec{\varphi} \mathrm{d} x \mathrm{~d} t \longrightarrow \int_{0}^{T} \int_{\mathbb{R}^{2}} \overline{\varrho \mathbf{U} \otimes \mathbf{U}}: \nabla_{x} \vec{\varphi} \mathrm{d} x \mathrm{~d} t,
$$

for any test function $\vec{\varphi} \in \mathscr{D}\left([0, T) ; \mathscr{D}\left(\mathbb{R}^{2} ; \mathbb{R}^{2}\right)\right)$, where $\overline{\varrho \mathbf{U} \otimes \mathbf{U}}$ means the weak limit of $\left\{\varrho_{\epsilon} \mathbf{u}_{\epsilon} \otimes \mathbf{u}_{\epsilon}\right\}_{\epsilon>0}$. To complete the proof, we need to show $\overline{\varrho \mathbf{U} \otimes \mathbf{U}}=\bar{\varrho} \mathbf{U} \otimes \mathbf{U}$ in the weak sense thanks to the oscillations of the gradient component of the velocity field and we postpone showing this part to handle the oscillations of the gradient component in the next section. To do this, we will use the estimate of the directional field $\left\{\mathbf{d}_{\epsilon}\right\}_{\epsilon>0}$ obtained in Jiang et al. [9]. We recall a basic estimate given in [10]. Consider the following.

Lemma 5. Let $c_{1}, c_{2}$ be positive numbers. Then there exists $C:=$ $C\left(c_{1}, c_{2}\right)$ such that if $\nabla \mathbf{d}_{\epsilon} \in W^{1,2}\left(\mathbb{R}^{2}\right)$ with $\left\|\nabla \mathbf{d}_{\epsilon}\right\|_{L^{2}\left(\mathbb{R}^{2}\right)} \leq c_{1}$ and $d_{2} \geq c_{2}$, then one has

$$
\left\|\Delta \mathbf{d}_{\epsilon}\right\|_{L^{2}\left(\mathbb{R}^{2}\right)}^{2}+\left\|\nabla \mathbf{d}_{\epsilon}\right\|_{L^{4}\left(\mathbb{R}^{2}\right)}^{2} \leq C\left\|\Delta \mathbf{d}_{\epsilon}+\left|\nabla \mathbf{d}_{\epsilon}\right|^{2} \mathbf{d}_{\epsilon}\right\|_{L^{2}\left(\mathbb{R}^{2}\right)}^{2} .
$$


To apply the Aubin-Lions lemma, let us compute the uniform estimate of $\partial_{t} \mathbf{d}_{\epsilon}$ with (5) and it follows that

$$
\begin{aligned}
\| \partial_{t} \mathbf{d}_{\epsilon} & \|_{L^{4 / 3}\left(0, T ; L^{2}(K)\right)} \\
\leq & \left\|\mathbf{u}_{\epsilon} \cdot \nabla \mathbf{d}_{\epsilon}\right\|_{L^{4 / 3}\left(0, T ; L^{2}(K)\right)} \\
& +\left\|\theta_{\epsilon}\left(\Delta \mathbf{d}_{\epsilon}+\left|\nabla \mathbf{d}_{\epsilon}\right|^{2} \mathbf{d}_{\epsilon}\right)\right\|_{L^{4 / 3}\left(0, T ; L^{2}(K)\right)} \\
\leq & C_{1}(K)\left\|\nabla \mathbf{u}_{\epsilon}\right\|_{L^{2}((0, T) \times K)} \mid\left\|\nabla \mathbf{d}_{\epsilon}\right\|_{L^{4}((0, T) \times K)} \\
& +C_{2}\left\|\Delta \mathbf{d}_{\epsilon}+\left|\nabla \mathbf{d}_{\epsilon}\right|^{2} \mathbf{d}_{\epsilon}\right\|_{L^{2}((0, T) \times K)}^{2} \\
\leq & C_{3}(K) \quad \text { for any compact } K \subset \subset \mathbb{R}^{2},
\end{aligned}
$$

where we have used Poincare's inequality, Holder's inequality, and estimate (27). Applying Aubin-Lions lemma together with (40) implies

$$
\mathbf{d}_{\epsilon} \longrightarrow \mathbf{d} \text { strongly } L^{2}\left(0, T ; W^{1,2}(K)\right),
$$

for any $K \subset \subset \mathbb{R}^{2}$. Thus we get

$$
\nabla \mathbf{d}_{\epsilon} \otimes \nabla \mathbf{d}_{\epsilon}-\frac{1}{2}\left|\nabla \mathbf{d}_{\epsilon}\right|^{2} \square \longrightarrow \nabla \mathbf{d} \otimes \nabla \mathbf{d}-\frac{1}{2}|\nabla \mathbf{d}|^{2} \llbracket,
$$

in the sense of distribution and $|\mathbf{d}|=1$. Consequently, using the estimates of $\left\{\mathbf{d}_{\epsilon}\right\}_{\epsilon>0}$ implies that

$$
\begin{aligned}
& \int_{0}^{T} \int_{\mathbb{R}^{2}} \mathbf{d} \cdot \partial_{t} \vec{\varphi} \mathrm{d} x \mathrm{~d} t \\
& +\int_{0}^{T} \int_{\mathbb{R}^{2}}\left((\mathbf{U} \cdot \mathbf{d}) \operatorname{div}_{x} \vec{\varphi}+(\nabla \mathbf{U} \cdot \mathbf{d}) \cdot \vec{\varphi}\right. \\
& \left.+\theta\left(\Delta \mathbf{d}+|\nabla \mathbf{d}|^{2} \mathbf{d}\right) \cdot \vec{\varphi}\right) \mathrm{d} x \mathrm{~d} t \\
& =-\int_{\mathbb{R}^{2}} \mathbf{d}_{0} \cdot \vec{\varphi}(0, x) \mathrm{d} x,
\end{aligned}
$$

for all $\vec{\varphi} \in\left[\mathscr{D}\left([0, T) \times \mathbb{R}^{2}\right)\right]^{2}$.

We are now able to identify the limit problem of the moment equation (11). Let us take the limit $\epsilon \rightarrow 0$ in the moment equation (11) and we get

$$
\begin{aligned}
\int_{0}^{T} & \int_{\mathbb{R}^{2}}\left(\bar{\varrho} \mathbf{U} \cdot \partial_{t} \varphi+\overline{\varrho \mathbf{U} \otimes \mathbf{U}}: \nabla_{x} \varphi\right) \mathrm{d} x \mathrm{~d} t \\
= & \int_{0}^{T} \int_{\mathbb{R}^{2}}\left(\mu \nabla_{x} \mathbf{U}: \nabla_{x} \varphi-\nu(\nabla \mathbf{d} \otimes \nabla \mathbf{d}): \nabla_{x} \varphi\right) \mathrm{d} x \mathrm{~d} t \\
& -\int_{\mathbb{R}^{2}}(\bar{\varrho} \mathbf{U})_{0} \cdot \varphi \mathrm{d} x
\end{aligned}
$$

for any test function

$$
\varphi \in C_{c}^{\infty}\left((0, T) \times \mathbb{R}^{2} ; \mathbb{R}^{3}\right), \quad \operatorname{div}_{x} \varphi=0,
$$

where we have assumed

$$
\mathbf{u}_{0, \epsilon} \longrightarrow \mathbf{U}_{0} \quad \text { weakly in } L^{2}\left(\mathbb{R}^{2} ; \mathbb{R}^{2}\right) .
$$

In general we do not expect

$$
\overline{\varrho \mathbf{U} \otimes \mathbf{U}}=\bar{\varrho} \mathbf{U} \otimes \mathbf{U},
$$

but our aim is to show that (47) holds in the weak sense; namely,

$$
\int_{0}^{T} \int_{\mathbb{R}^{2}} \overline{\varrho \mathbf{U} \otimes \mathbf{U}}: \nabla_{x} \varphi \mathrm{d} x \mathrm{~d} t=\int_{0}^{T} \int_{\mathbb{R}^{2}}[\bar{\varrho} \mathbf{U} \otimes \mathbf{U}]: \nabla_{x} \varphi \mathrm{d} x \mathrm{~d} t
$$

for any

$$
\varphi \in C_{c}^{\infty}\left((0, T) \times \mathbb{R}^{2} ; \mathbb{R}^{2}\right), \quad \operatorname{div}_{x} \varphi=0 .
$$

3.4. Strong Convergence of the Velocity. In this section, our aim is to show that (48) holds. To do this we will follow the framework of Feireisl [7]. Indeed, it is sufficient to show that

$$
\mathbf{u}_{\epsilon} \longrightarrow \mathbf{U} \text { strongly in } L^{2}((0, T) \times K), K \subset \subset \mathbb{R}^{2}
$$

which is equivalent to the following convergence:

$$
\begin{aligned}
& {\left[t \longmapsto \int_{\mathbb{R}^{2}} \mathbf{V}_{\epsilon}(t, \cdot) \cdot \phi \mathrm{d} x\right]} \\
& \quad \longrightarrow\left[t \longmapsto \int_{\mathbb{R}^{2}} \mathbf{V}(t, \cdot) \cdot \phi \mathrm{d} x\right] \quad \text { in } L^{1}(0, T)
\end{aligned}
$$

for any $\phi \in C_{c}^{\infty}\left(K ; \mathbb{R}^{2}\right)$, where $\mathbf{V}_{\epsilon}=\varrho_{\epsilon} \mathbf{u}_{\epsilon}$, and we have here used the embedding $W^{1,2}\left(\mathbb{R}^{2}\right) \hookrightarrow L^{2}(K)$ for any compact subset $K \subset \subset \mathbb{R}^{2}$ and the convergence of (37). For the proof, we will apply Kato's result [6], Theorem 6.

Theorem 6 (see Reed and Simon [11]). Let A be a closed densely defined linear operator and $H$ a self-adjoint densely defined operator in a Hilbert space $M$. For $\lambda \in \mathbb{C}-\mathbb{R}$, let $R_{H}[\lambda]=(H-\lambda I d)^{-1}$ denote the resolvent of $H$. Suppose that

$$
\Gamma:=\sup _{\lambda \in \mathbb{C}-\mathbb{R}, v \in \mathscr{D}\left(A^{*}\right),\|v\|=1}\left\|A \circ R_{H}[\lambda] \circ A^{*}[v]\right\|_{X}<\infty .
$$

Then

$$
\sup _{w \in X,\|w\|_{X}=1} \frac{\pi}{2} \int_{-\infty}^{\infty}\|A \exp (-i t H)[w]\|_{X}^{2} d t \leq \Gamma^{2} .
$$

We next study the acoustic equations. The acoustic equations are used to describe the time evolution of fast acoustic waves in the compressible models in order to handle the oscillation of $\mathbf{Q}\left[\varrho_{\epsilon} \mathbf{u}_{\epsilon}\right]$. To begin with, we rewrite the equations in (5):

$$
\int_{0}^{T} \int_{\mathbb{R}^{2}}\left(\epsilon X_{\epsilon} \partial_{t} \varphi+\mathbf{V}_{\epsilon} \cdot \nabla_{x} \varphi\right) \mathrm{d} x \mathrm{~d} t=0,
$$

for any $\varphi \in C_{c}^{\infty}\left((0, T) \times \mathbb{R}^{2}\right)$ and

$$
\begin{gathered}
\int_{0}^{T} \int_{\mathbb{R}^{2}}\left(\epsilon \mathbf{V}_{\epsilon} \cdot \partial_{t} \psi+\omega X_{\epsilon} \operatorname{div}_{x} \psi\right) \mathrm{d} x \mathrm{~d} t \\
=\epsilon \int_{0}^{T} \int_{\mathbb{R}^{2}} \Pi_{\epsilon}: \nabla_{x} \psi \mathrm{d} x \mathrm{~d} t,
\end{gathered}
$$


for any $\psi \in C_{c}^{\infty}\left((0, T) \times \mathbb{R}^{2}\right)$, where

$$
\begin{gathered}
X_{\epsilon}=\frac{\varrho_{\epsilon}-\bar{\varrho}}{\epsilon}, \quad \mathbf{V}_{\epsilon}=\varrho_{\epsilon} \mathbf{u}_{\epsilon}, \quad \omega=\gamma \bar{\varrho}^{\gamma-1}, \\
\Pi_{\epsilon}=\mu_{\epsilon} \nabla \mathbf{u}_{\epsilon}-\left(\varrho_{\epsilon} \mathbf{u}_{\epsilon} \otimes \mathbf{u}_{\epsilon}\right)-\nu_{\epsilon}\left(\nabla \mathbf{d}_{\epsilon} \otimes \nabla \mathbf{d}_{\epsilon}\right) \\
+\left(\frac{1}{2}\left|\nabla \mathbf{d}_{\epsilon}\right|^{2}-\left(\left(\varrho_{\epsilon}^{\gamma}-\bar{\varrho}^{\gamma}\right)-\gamma \bar{\varrho}^{\gamma-1}\left(\varrho_{\epsilon}-\bar{\varrho}\right)\right)\right)
\end{gathered}
$$

and $\Pi_{\epsilon}$ can be written by

$$
\Pi_{\epsilon}=\Pi_{\epsilon}^{1}+\Pi_{\epsilon}^{2}
$$

satisfying

$$
\begin{aligned}
& \left\{\Pi_{\epsilon}^{1}\right\}_{\epsilon>0} \text { bounded in } L^{2}\left(0, T ; L^{1}\left(\mathbb{R}^{2} ; \mathbb{R}^{2 \times 2}\right)\right), \\
& \left\{\Pi_{\epsilon}^{2}\right\}_{\epsilon>0} \text { bounded in } L^{2}\left(0, T ; L^{2}\left(\mathbb{R}^{2} ; \mathbb{R}^{2 \times 2}\right)\right) .
\end{aligned}
$$

We now introduce the Helmholtz decomposition and the following material may be found in most of the text books of fluid mechanics.

Theorem 7. A vector function $\mathbf{v}: \mathbb{R}^{2} \rightarrow \mathbb{R}^{2}$ is written as

$$
\mathbf{v}=\mathbf{P}[\mathbf{v}]+\mathbf{Q}[\mathbf{v}],
$$

where

$$
\mathbf{Q}[\mathbf{v}]=\nabla_{x} \Phi, \quad \Delta \Phi=\operatorname{div}_{x} \mathbf{v} \text { in } \Omega, \int_{\mathbb{R}^{2}} \Phi d x=0 .
$$

We now apply the Helmholtz projection to (54) and (55) in order to obtain (51) with the test function $\psi=\nabla \Psi$ verifying

$$
\operatorname{div}_{x}(\nabla \Psi)=\chi \in C_{c}^{\infty}\left([0, T) \times \mathbb{R}^{2}\right), \quad \Psi(t, \cdot) \in \mathscr{D}^{1,2}\left(\mathbb{R}^{2}\right),
$$

such that the initial value problem (54) and (55) can be written as follows:

$$
\int_{0}^{T} \int_{\mathbb{R}^{2}}\left(\epsilon X_{\epsilon} \partial_{t} \varphi+\nabla_{x} \Phi_{\epsilon} \cdot \nabla \varphi\right) \mathrm{d} x \mathrm{~d} t=-\epsilon \int_{\mathbb{R}^{2}} X_{0, \epsilon} \varphi(0, \cdot) \mathrm{d} x,
$$

for any $\varphi \in C_{c}^{\infty}\left([0, T) \times \mathbb{R}^{2}\right)$ where $\nabla \Phi_{\epsilon}=\mathbf{Q}\left[\mathbf{V}_{\epsilon}\right]$ and

$$
\begin{aligned}
& \int_{0}^{T} \int_{\mathbb{R}^{2}}\left(-\epsilon \Phi_{\epsilon} \partial_{t} \chi+\omega X_{\epsilon} \chi\right) \mathrm{d} x \mathrm{~d} t \\
& =\epsilon \int_{\mathbb{R}^{2}} \Phi_{0, \epsilon} \chi(0, \cdot) \mathrm{d} x \\
& \quad+\epsilon \int_{0}^{T} \int_{\mathbb{R}^{2}}\left(\left(\Pi_{\epsilon}^{1}+\Pi_{\epsilon}^{2}\right): \nabla^{2} \Phi_{\epsilon}\right) \mathrm{d} x \mathrm{~d} t .
\end{aligned}
$$

We rewrite the system (63) in a single differential operator in order to apply Theorem 6 in the space $L^{2}\left(\mathbb{R}^{2}\right)$. Denote some notations by

$$
\mathscr{D}(\Delta)=\left\{g \in L^{2}(\Omega) \mid g \in W_{0}^{2,2}\left(\mathbb{R}^{2}\right)\right\}
$$

and $G(-\Delta)[v]$ by

$$
\begin{array}{r}
\langle G(-\Delta)[v], \varphi\rangle=\langle v, G(-\Delta)[\varphi]\rangle \\
\quad=\int_{0}^{\infty} G(\lambda) \mathrm{d}\left(\int_{\Omega} P_{\lambda}[\bar{\varphi}] v \mathrm{~d} x\right),
\end{array}
$$

for $\varphi \in C_{c}^{\infty}\left(\mathbb{R}^{2}\right)$ and the spectral resolution associated with the operator $-\Delta$ in $L^{2}\left(\mathbb{R}^{2}\right)$. We show that, for given $\Phi, \chi$ in (61),

$$
\chi \longmapsto \int_{\mathbb{R}^{2}}\left(\Pi_{\epsilon}^{1}+\Pi_{\epsilon}^{2}\right): \nabla^{2} \Psi \mathrm{d} x
$$

is a bounded linear functional for

$$
\chi \in \mathscr{D}(-\Delta) \cap \mathscr{D}\left(\frac{1}{\sqrt{-\Delta}}\right),
$$

by the norm estimates of $\left\|\Pi_{\epsilon}^{1}\right\|_{L^{1}\left(\mathbb{R}^{2} ; \mathbb{R}^{2 \times 2}\right)},\left\|\Pi_{\epsilon}^{2}\right\|_{L^{2}\left(\mathbb{R}^{2} ; \mathbb{R}^{2 \times 2}\right)}$. We apply the Riezs representation theorem to the system (62) and (63) by putting $\omega=1$ in order to obtain the following system:

$$
\begin{aligned}
& \int_{0}^{T} \int_{\mathbb{R}^{2}}\left(\epsilon X_{\epsilon} \partial_{t} \chi+\sqrt{-\Delta}\left[\Phi_{\epsilon}\right] \sqrt{-\Delta}[\chi]\right) \mathrm{d} x \mathrm{~d} t \\
& \quad=-\epsilon \int_{\mathbb{R}^{2}} X_{0, \epsilon} \chi(0, \cdot) \mathrm{d} x,
\end{aligned}
$$

for all $\chi \in C_{c}^{\infty}\left([0, T) ; \mathscr{D}\left(\mathbb{R}^{2}\right)\right)$ and

$$
\begin{aligned}
& \int_{0}^{T} \int_{\mathbb{R}^{2}}\left(-\epsilon \Phi_{\epsilon} \partial_{t} \chi+X_{\epsilon} \chi\right) \mathrm{d} x \mathrm{~d} t \\
& =\epsilon \int_{\mathbb{R}^{2}} \Phi_{0, \epsilon} \chi(0, \cdot) \mathrm{d} x \\
& \quad+\epsilon \int_{0}^{T} \int_{\mathbb{R}^{2}}\left(k_{\epsilon}^{1} \Delta[\chi]+k_{\epsilon}^{2} \frac{1}{\sqrt{-\Delta}}[\chi]\right) \mathrm{d} x \mathrm{~d} t,
\end{aligned}
$$

for any test function

$$
\chi \in C_{c}^{\infty}\left([0, T) ; \mathscr{D}(\Delta) \cap \mathscr{D}\left(\frac{1}{\sqrt{-\Delta}}\right)\right),
$$

$\left\{k_{\epsilon}^{i}\right\} \quad$ bounded in $L^{2}\left((0, T) \times \mathbb{R}^{2}\right), i=1,2$.

Consequently, the system (68) and (69) can formally be written into

$$
\begin{gathered}
\epsilon \partial_{t} \Phi_{\epsilon}+X_{\epsilon}=\epsilon\left(\Delta\left[k_{\epsilon}^{1}\right]+\frac{1}{\sqrt{-\Delta}}\left[k_{\epsilon}^{2}\right]\right), \\
\epsilon \partial_{t} X_{\epsilon}+\Delta\left[\Phi_{\epsilon}\right]=0,
\end{gathered}
$$

with the initial conditions $X_{\epsilon}(0, \cdot)=X_{0, \epsilon}, \Phi_{\epsilon}(0, \cdot)=\Phi_{0, \epsilon}$. 
From Duhamel's formula solving the initial boundary value problem (71), we get

$$
\begin{aligned}
\Phi_{\epsilon}(t)= & \exp \left(\mathrm{i} \frac{t}{\epsilon} \sqrt{-\Delta}\right)\left[\frac{1}{2} \Phi_{0, \epsilon}+\frac{\mathrm{i}}{2 \sqrt{-\Delta}}\left[X_{0, \epsilon}\right]\right] \\
& +\exp \left(-\mathrm{i} \frac{t}{\epsilon} \sqrt{-\Delta}\right)\left[\frac{1}{2} \Phi_{0, \epsilon}-\frac{\mathrm{i}}{2 \sqrt{-\Delta}}\left[X_{0, \epsilon}\right]\right] \\
& +\int_{0}^{t} \exp \left(\mathrm{i} \frac{t-s}{\epsilon} \sqrt{-\Delta}\right) \\
& \cdot\left[\Delta\left[k_{\epsilon}^{1}(s, \cdot)\right]+\frac{1}{\sqrt{-\Delta}}\left[k_{\epsilon}^{2}(s, \cdot)\right]\right] \mathrm{d} s .
\end{aligned}
$$

Duhmel's formula (72) can rigorously be represented by

$$
\begin{aligned}
& \left\langle\Phi_{\epsilon}(t, \cdot), G(-\Delta)(\chi)\right\rangle \\
& =\frac{1}{2}\left\langle G(-\Delta)\left[\exp \left(i \frac{t}{\epsilon} \sqrt{-\Delta}\right)+\exp \left(-\mathrm{i} \frac{t}{\epsilon} \sqrt{-\Delta}\right)\right]\right. \\
& \left.\cdot[\chi], \phi_{0, \epsilon}\right\rangle \\
& +\frac{1}{2}\left\langle\frac{\mathrm{i} G(-\Delta)}{\sqrt{-\Delta}}\left[\exp \left(\mathrm{i} \frac{t}{\epsilon} \sqrt{-\Delta_{N}}\right)-\exp \left(-\mathrm{i} \frac{t}{\epsilon} \sqrt{-\Delta}\right)\right]\right. \\
& +\int_{0}^{t}\left\langle\Delta G(-\Delta) \cos \left(\frac{t-s}{\epsilon} \sqrt{-\Delta}\right)[\chi], k_{\epsilon}^{1}(s, \cdot)\right\rangle \mathrm{d} s \\
& +\int_{0}^{t}\left\langle\frac{G(-\Delta)}{\sqrt{-\Delta}} \cos \left(\frac{t-s}{\epsilon} \sqrt{-\Delta}\right)[\chi], k_{\epsilon}^{2}(s, \cdot)\right\rangle \mathrm{d} s,
\end{aligned}
$$

for any $G \in C_{c}^{\infty}(0, \infty)$.

In the sprite of Feireisl [7], we can prove (51) for the gradient part $\mathbf{Q}\left[\mathbf{V}_{\epsilon}\right]$ together with applying Theorem 6 with $M=L^{2}\left(\mathbb{R}^{2}\right), H=\sqrt{-\Delta}, A=\varphi G(-\Delta), \varphi \in C_{c}^{\infty}\left(\mathbb{R}^{2}\right)$, and $G \in C_{c}^{\infty}(0, \infty)$.

\section{Conflict of Interests}

The author declares that there is no conflict of interests regarding the publication of this paper.

\section{Acknowledgment}

The work of the author was supported by the Basic Science Research Program through the National Research Foundation of Korea (NRF) funded by the Ministry of Education (NRF-2013R1A1A2057662).

\section{References}

[1] S. Klainerman and A. Majda, "Singular limits of quasilinear hyperbolic systems with large parameters and the incompressible limit of compressible fluids," Communications on Pure and Applied Mathematics, vol. 34, no. 4, pp. 481-524, 1981.
[2] P.-L. Lions and N. Masmoudi, "Incompressible limit for a viscous compressible fluid," Journal de Mathématiques Pures et Appliquées, vol. 77, no. 6, pp. 585-627, 1998.

[3] E. Feireisl and A. Novotný, Singular Limits in Thermodynamics of Viscous Fluids, Advances in Mathematical Fluid Mechanics, Birkhäuser, Basel, Switzerland, 2009.

[4] E. Feireisl and A. Novotný, "The low Mach number limit for the full Navier-Stokes-Fourier system," Archive for Rational Mechanics and Analysis, vol. 186, no. 1, pp. 77-107, 2007.

[5] D. Wang and C. Yu, "Incompressible limit for the compressible flow of liquid crystals," Journal of Mathematical Fluid Mechanics, vol. 16, no. 4, pp. 771-786, 2014.

[6] T. Kato, "Wave operators and similarity for some non-selfadjoint operators," Mathematische Annalen, vol. 162, no. 2, pp. 258-279, 1966.

[7] E. Feireisl, "Incompressible limits and propagation of acoustic waves in large domains with boundaries," Communications in Mathematical Physics, vol. 294, no. 1, pp. 73-95, 2010.

[8] Y.-S. Kwon and K. Trivisa, "On the incompressible limits for the full magnetohydrodynamics flows," Journal of Differential Equations, vol. 251, no. 7, pp. 1990-2023, 2011.

[9] F. Jiang, S. Jiang, and D. Wang, "Global weak solutions to the equations of compressible flow of nematic liquid crystals in two dimensions," Archive for Rational Mechanics and Analysis, vol. 214, no. 2, pp. 403-451, 2014.

[10] Z. Lei, D. Li, and X.-Y. Zhang, "Remarks of global wellposedness of liquid crystal flows and heat flows of harmonic maps in two dimensions," Proceedings of the American Mathematical Society, vol. 142, no. 11, pp. 3801-3810, 2014.

[11] M. Reed and B. Simon, Methods of Modern Mathematical Physics. IV. Analysis of Operator, Academy Press (Harcourt Brace Jovanovich Publishers), New York, NY, USA, 1978. 


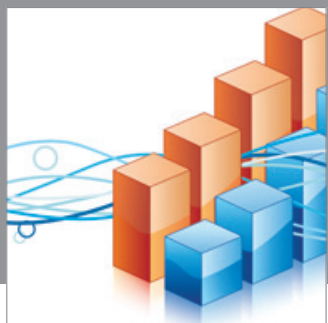

Advances in

Operations Research

mansans

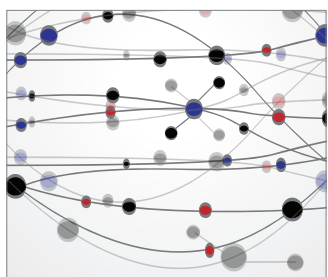

The Scientific World Journal
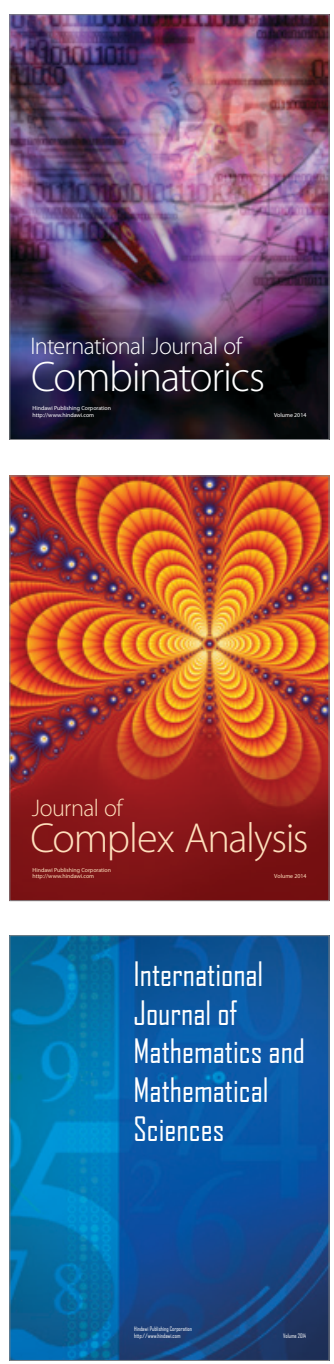
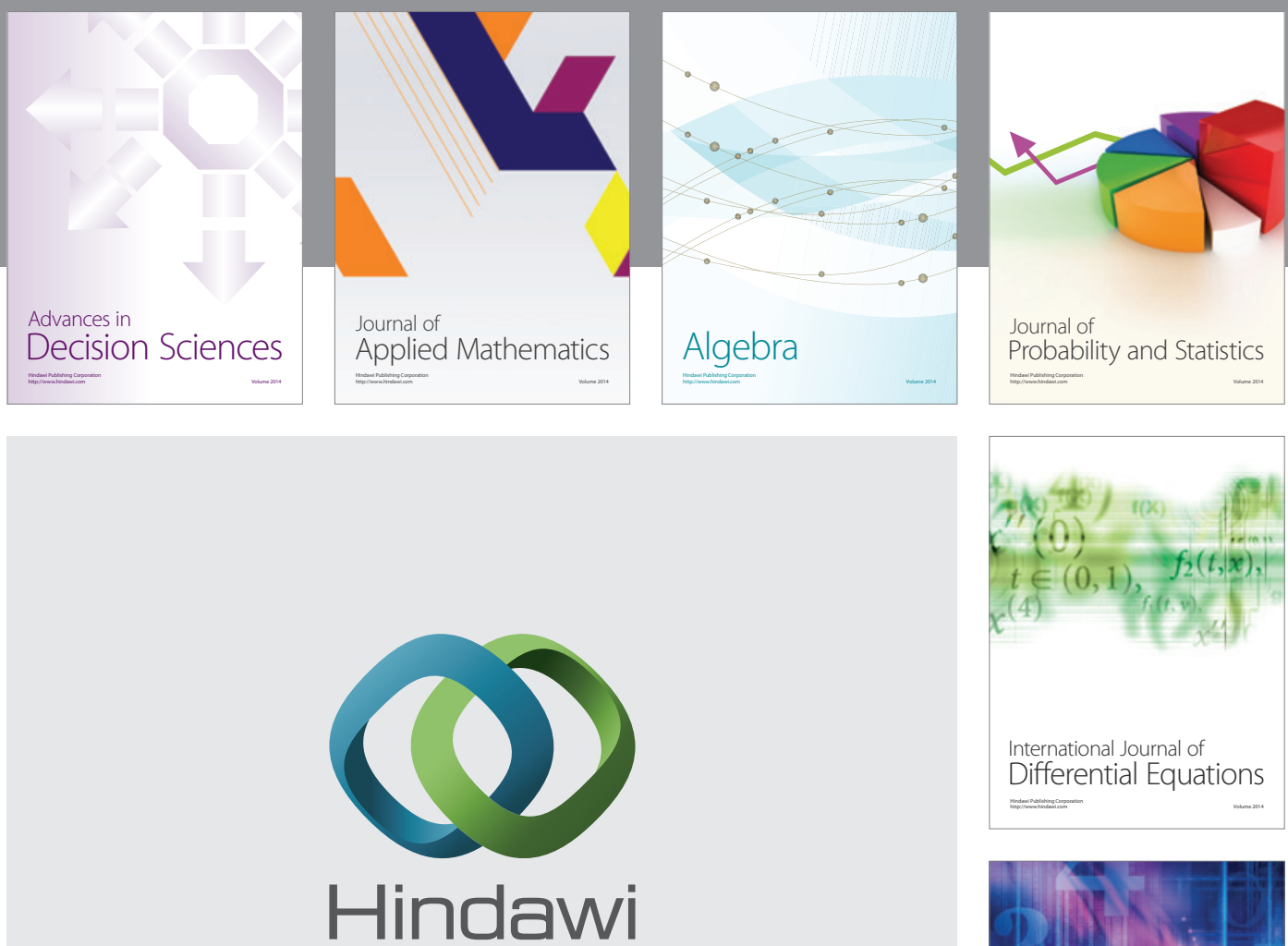

Submit your manuscripts at http://www.hindawi.com
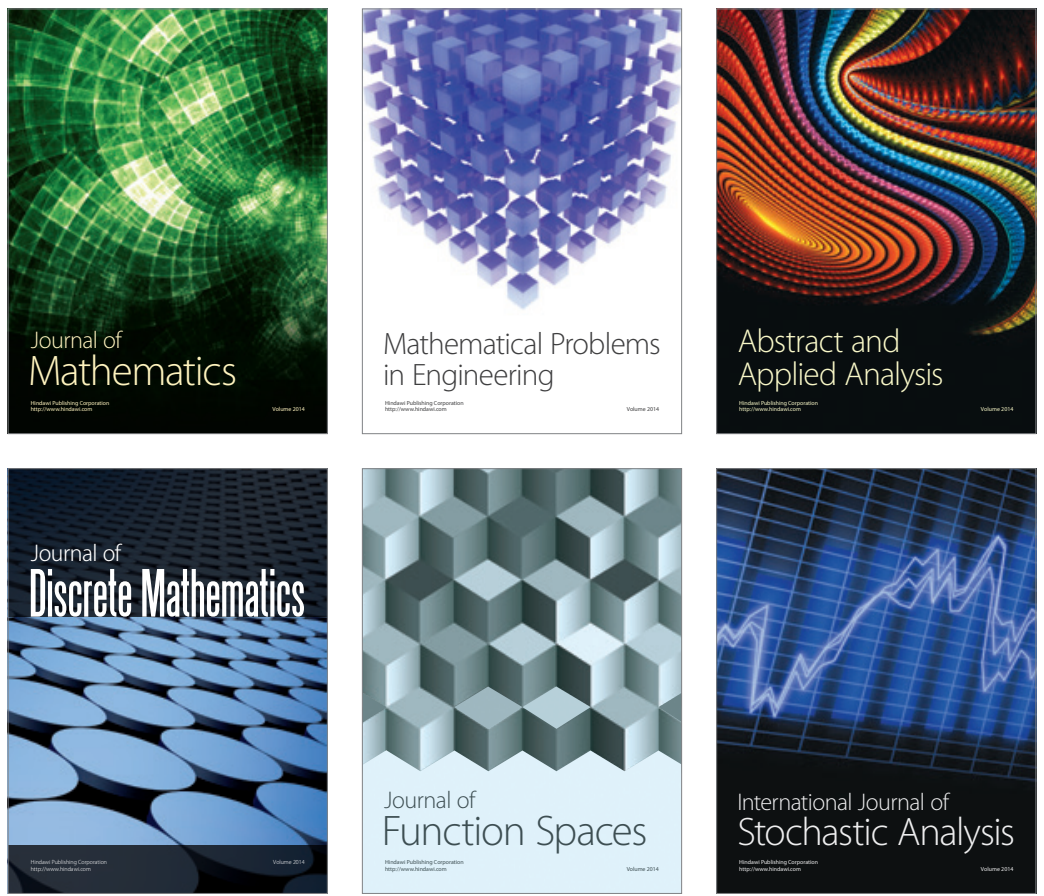

Journal of

Function Spaces

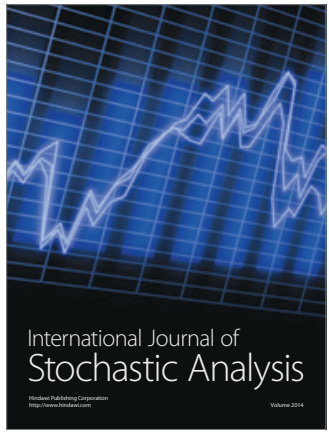

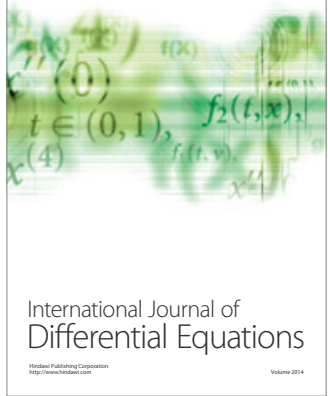
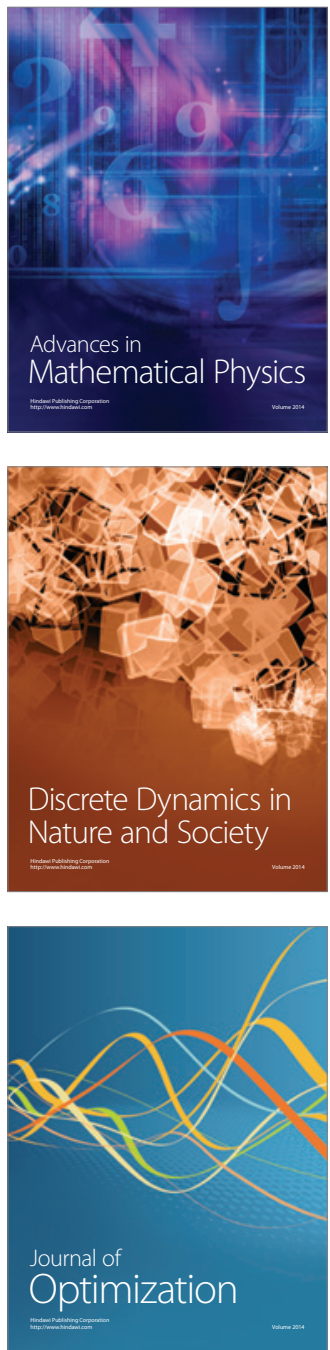